ANNALES

UNIVERSITATIS MARIAE CURIE-SKŁODOWSKA

L UBLIN - POLONIA

\title{
Trace parameters for Teichmüller space of genus 2 surfaces and mapping class group
}

\begin{abstract}
We obtain a representation of the mapping class group of genus 2 surface in terms of a coordinate system of the Teichmüller space defined by trace functions.
\end{abstract}

1. Introduction. We identify $P S L(2, \mathbb{R})$ with the group of orientationpreserving isometries of the upper half plane $\mathbb{H}=\{z \in \mathbb{C}: \operatorname{Im} z>0\}$ equipped with the hyperbolic metric $|d z| /(\operatorname{Im} z)$.

A Fuchsian subgroup $G$ of $P S L(2, \mathbb{R})$ is said to be of type $(2 ;-;-;-)$ ([5, p. 38]) if $\mathbb{H} / G$ is a closed surface of genus 2 and the projection $\pi: \mathbb{H} \rightarrow \mathbb{H} / G$ is an unbranched covering. $G$ has a canonical generator system or a marking $E=(A, B, C, D)$ which satisfies

$$
[A, B][C, D]=1,
$$

where $[a, b]=a b a^{-1} b^{-1}$ is the commutator of $a$ and $b$, and 1 stands for the unit matrix. We call the pair $(G, E)$ a marked Fuchsian group of type $(2 ;-;-;-)$. Two marked Fuchsian groups $\left(G_{1}, E_{1}\right)$ and $\left(G_{2}, E_{2}\right)$ are equivalent if there exists a matrix $P \in P S L(2, \mathbb{R})$ such that

$$
A_{2}=P^{-1} A_{1} P, B_{2}=P^{-1} B_{1} P, C_{2}=P^{-1} C_{1} P, D_{2}=P^{-1} D_{1} P,
$$

2010 Mathematics Subject Classification. Primary 32G15; Secondary 30F35.

Key words and phrases. Teichmüller space, Fuchsian group, mapping class group.

The first author was supported by JSPS KAKENHI Grant Number 20740081 and the second author was supported by JSPS KAKENHI Grant Number 22540191. 
where $E_{j}=\left(A_{j}, B_{j}, C_{j}, D_{j}\right), j=1,2$. The Teichmüller space $\mathcal{T}_{2}$ of type $(2 ;-;-;-)$ is the space of all equivalence classes of marked Fuchsian groups of type $(2 ;-;-;-)$. Each marked Fuchsian group $(G, E)$ can be represented by a tuple $(A, B, C, D)$ of matrices in $S L(2, \mathbb{R})$ such that

$$
\operatorname{tr} A>0, \operatorname{tr} B>0, \operatorname{tr} C>0 \text { and } \operatorname{tr} D>0 .
$$

Therefore, for the rest of this paper, we always assume that $E=(A, B, C, D)$ consists of matrices satisfying (1.1). In this case $\operatorname{tr} A B$ and $\operatorname{tr} C D$ are both positive (this follows from $[5,33.17(\mathrm{~b})]$ ). In [3] we considered the following traces as functions of $\left[(G, E=(A, B, C, D)]\right.$ in $\mathcal{T}_{2}$ :

$$
\begin{aligned}
& a=\operatorname{tr} A, b=\operatorname{tr} B, z=\operatorname{tr} A B, u=-\operatorname{tr} A C D C^{-1}, \\
& v=-\operatorname{tr} A C D^{2}, w=-\operatorname{tr} A C D, t=\operatorname{tr} C D .
\end{aligned}
$$

Since all non trivial elements of $G$ are hyperbolic, their traces take values in $\mathbb{R}_{>2}=\{x: x>2\}$. It is shown in [3] (see also [4]) that the mapping $\Phi: \mathcal{T}_{2} \rightarrow \mathbb{R}_{>2}^{7}$ defined by $\Phi([G, E])=(a, b, z, u, v, w, t)$ is an embedding and $a, b, z, u, v, w, t$ satisfy the identity

$$
a w t+a^{2}+w^{2}+t^{2}+K^{2}+S^{2}+4-w \sqrt{\left(K^{2}+4\right)\left(S^{2}+4\right)}=0,
$$

where

$$
K=\sqrt{a b z-a^{2}-b^{2}-z^{2}} \text { and } S=\sqrt{u v t-u^{2}-v^{2}-t^{2}} .
$$

The mapping class group $\mathcal{M} C_{2}$ is the group of isotopy classes of orientation-preserving homeomorphisms of the orientable closed surface $S$ of genus 2. It is a subgroup of outer automorphisms of the fundamental group of $S$ (see [5]). $\mathcal{M} C_{2}$ acts on the Teichmüller space $\mathcal{T}_{2}$ by changing the marking. The purpose of this paper is to describe a generating system of $\mathcal{M} C_{2}$ by using the coordinate-system $(a, b, z, u, v, w, t)$. It is an interesting observation that $\mathcal{M} C_{2}$ acts on $\mathcal{T}_{2}$ as a group of rational transformations.

\section{Trace identities.}

2.1. Basic trace identities. The matrices $A, B$ and $C$ in $S L(2, \mathbb{R})$ satisfy the following identities (see $[2, \S 3.4]$ ):

(I1) $\operatorname{tr} A=\operatorname{tr} A^{-1}$

(I2) $\operatorname{tr} A B+\operatorname{tr} A B^{-1}=\operatorname{tr} A \operatorname{tr} B$,

(I3) $\operatorname{tr} A B C=\operatorname{tr} A \operatorname{tr} B C+\operatorname{tr} B \operatorname{tr} C A+\operatorname{tr} C \operatorname{tr} A B-\operatorname{tr} A \operatorname{tr} B \operatorname{tr} C-\operatorname{tr} A C B$.

We shall use repeatedly the following identities, which are consequences of (I1), (I2) and (I3) above:

$$
\begin{aligned}
\operatorname{tr}[A, B] & =\operatorname{tr} A B A^{-1} B^{-1} \\
& =(\operatorname{tr} A)^{2}+(\operatorname{tr} B)^{2}+(\operatorname{tr} A B)^{2}-\operatorname{tr} A \operatorname{tr} B \operatorname{tr} A B-2, \\
\operatorname{tr} A B C B & =\operatorname{tr} A B \operatorname{tr} B C+\operatorname{tr} A C-\operatorname{tr} A \operatorname{tr} C \\
\operatorname{tr} A B C B^{-1} & =\operatorname{tr} A \operatorname{tr} C-\operatorname{tr} A C-\operatorname{tr} A B \operatorname{tr} B C+\operatorname{tr} B \operatorname{tr} A B C .
\end{aligned}
$$


Let $G$ be a group generated by a finite number of matrices $A_{1}, \ldots, A_{n} \in$ $S L(2, \mathbb{R})$ and

$$
\mathcal{S}=\left\{\operatorname{tr}\left(A_{i_{1}} A_{i_{2}} \cdots A_{i_{r}}\right): 1 \leq i_{1}<i_{2}<\cdots<i_{r} \leq n, 1 \leq r \leq n\right\} .
$$

Then the following fact is well known (see $[2, \S 3.5]$ ).

Lemma 2.1. Let $g \in G$. Then $\operatorname{tr} g$ is an integer polynomial in $\mathcal{S}$.

2.2. Trace identities for genus 2 surface. Let $E=(A, B, C, D)$ be a marking of a Fuchsian group $G$ of type $(2 ;-;-;-)$. Let $c=x_{1}=\operatorname{tr} C$ and $d=x_{2}=\operatorname{tr} D, x_{3}=\operatorname{tr} A C, x_{4}=\operatorname{tr} A D, x_{5}=\operatorname{tr} B C, x_{6}=\operatorname{tr} B D$, $x_{7}=\operatorname{tr} A B C, x_{8}=\operatorname{tr} A B D, x_{9}=\operatorname{tr} B C D$ and $x_{10}=\operatorname{tr} A B C D$. Then the set $\mathcal{S}$ for $G$ with respect to $(A, B, C, D)$ is

$$
\mathcal{S}=\left\{a, b, c, d, z, x_{3}, x_{4}, x_{5}, x_{6}, t, x_{7}, x_{8}, x_{9}, x_{10}\right\} .
$$

The purpose of this section is to find expressions of $x_{1}, \ldots, x_{10}$ in $\{a, b, z, u, v$, $w, t\}$ of (1.2). Then by Lemma 2.1 we can express the trace of any element of $G$ in $\{a, b, z, u, v, w, t\}$. We shall apply this fact to obtain a representation of the mapping class group $\mathcal{M} C_{2}$ via rational transformations.

(1) Since $[A, B]=[C, D]^{-1}$, we obtain by (2.1a)

$$
a b z-a^{2}-b^{2}-z^{2}=c d t-c^{2}-d^{2}-t^{2} .
$$

Note that $\operatorname{tr}[A, B]=a^{2}+b^{2}+z^{2}-a b z-2<-2$, since $G$ is discrete (see, for example [5, $33 \mathrm{D}])$. In what follows $K=\sqrt{a b z-a^{2}-b^{2}-z^{2}}$.

(2) From $B A B^{-1}=C D C^{-1} D^{-1} A$ and the basic identity (I3) we obtain

$$
a=\operatorname{tr}\left((A C D) \cdot C^{-1} \cdot D^{-1}\right)=-w t+c x_{3}-u d+w c d-a .
$$

and hence

$$
2 a+w t-c x_{3}+u d-w c d=0 .
$$

(3) From (I2), $v=-\operatorname{tr} A C D \cdot D=-(\operatorname{tr} A C D \operatorname{tr} D-\operatorname{tr} A C)=w d+x_{3}$ and so

$$
x_{3}=v-d w .
$$

From this and (2.4) it follows that

$$
2 a+w t-c v+u d=0 .
$$

(4) From (I3),

$$
\begin{aligned}
-u & =\operatorname{tr} A \cdot C D \cdot C^{-1}=a d+t\left(\operatorname{tr} A C^{-1}\right)-w c-a t c-x_{4} \\
& =a d+t\left(a c-x_{3}\right)-w c-a t c-x_{4} .
\end{aligned}
$$

It follows from this and (2.5) that

$$
x_{4}=u+a d-t x_{3}-w c=u+a d-t v+t w d-c w .
$$

By substituting $d=u^{-1}(c v-2 a-w t)$ (see (2.6)) into (2.3) we obtain

$$
\left(u v t-u^{2}-v^{2}\right) c^{2}-(2 a+w t)(t u-2 v) c-\left(K^{2}+t^{2}\right) u^{2}-(2 a+t w)^{2}=0 .
$$


If this identity is regarded as a quadratic equation in $c$, it always has a negative root because

$$
u v t-u^{2}-v^{2}=\left(-\operatorname{tr}\left[C D^{-1} C^{-1} A^{-1}, A C D^{2}\right]-2\right)+t^{2}>t^{2}>0
$$

$($ see $[5,33 \mathrm{D}])$ and $-\left(K^{2}+t^{2}\right) u^{2}-(2 a+t w)^{2}<0$. Hence the condition $c=\operatorname{tr} C>2$ yields

$$
\begin{aligned}
& c=\frac{(2 a+t w)(u t-2 v)+u \sqrt{(2 a+t w)^{2}\left(t^{2}-4\right)+4\left(K^{2}+t^{2}\right)\left(S^{2}+t^{2}\right)}}{2\left(S^{2}+t^{2}\right)}, \\
& d=\frac{c v-2 a-w t}{u}
\end{aligned}
$$

where $S=\sqrt{u v t-u^{2}-v^{2}-t^{2}}$. By using (1.3) we see that $(2 a+t w)^{2}\left(t^{2}-4\right)$ $+4\left(K^{2}+t^{2}\right)\left(S^{2}+t^{2}\right)$ equals

$$
\begin{aligned}
& \left(\left(t^{2}-4\right) w+2 \sqrt{\left(S^{2}+4\right)\left(K^{2}+4\right)}\right)^{2} \\
& \quad=\left(\left(t^{2}-4\right) w+\frac{2\left(a w t+a^{2}+t^{2}+K^{2}+S^{2}+4\right)}{w}\right)^{2} .
\end{aligned}
$$

Now from (2.8) we obtain

$$
\begin{aligned}
& c=\frac{\left(K^{2}+S^{2}+t^{2}+a^{2}+4\right) u+w\left(2 a t u-2 a v-u w+t^{2} u w-t v w\right)}{w\left(S^{2}+t^{2}\right)}, \\
& d=\frac{\left(K^{2}+S^{2}+t^{2}+a^{2}+4\right) v+w(2 a u+t w u-v w)}{w\left(S^{2}+t^{2}\right)} .
\end{aligned}
$$

By (2.5), (2.7) and (2.9), we can obtain the expressions of $x_{3}=\operatorname{tr} A C$ and $x_{4}=\operatorname{tr} A D$ in $(a, b, z, u, v, w, t)$,

$$
\begin{aligned}
& x_{3}=-\frac{u w(2 a+t w)+v\left(4+a^{2}+K^{2}-w^{2}\right)}{S^{2}+t^{2}} \\
& x_{4}=(a d+u-c w)+t \frac{\left(4+a^{2}+K^{2}-w^{2}\right) v+w u(2 a+t w)}{S^{2}+t^{2}} .
\end{aligned}
$$

(5) From (I2) and (2.1c) applied to $B C D C^{-1}$ we obtain

$$
\begin{aligned}
\operatorname{tr} B^{-1}\left(C D C^{-1}\right) & =b d-\operatorname{tr} B C D C^{-1} \\
& =b d-\left(b d-x_{6}-x_{5} t+c x_{9}\right)=x_{6}+t x_{5}-c x_{9} .
\end{aligned}
$$

From (I3), $\operatorname{tr} B^{-1} C D=b t-x_{9}$. Then, from the trace of $A B^{-1} A^{-1}=$ $B^{-1} C D \cdot C^{-1} \cdot D^{-1}$, (I2), (I3) and (2.11),

$$
\begin{aligned}
b & =\left(\operatorname{tr} B^{-1} C D\right) t+c \operatorname{tr} B^{-1} C+d \operatorname{tr}\left(B^{-1} C D \cdot C^{-1}\right)-\left(\operatorname{tr} B^{-1} C D\right) c d-b \\
& =\left(b t-x_{9}\right)(t-c d)+c\left(b c-x_{5}\right)+d\left(x_{6}+t x_{5}-c x_{9}\right)-b .
\end{aligned}
$$

Hence

$$
(d t-c) x_{5}+d x_{6}-t x_{9}=2 b-b t^{2}+b c d t-b c^{2} .
$$


(6) From (I2), $\operatorname{tr} A^{-1} C D=a t+w$, and from (I2) and (I3),

$$
\begin{aligned}
\operatorname{tr} B^{-1} A^{-1} \cdot C \cdot D & =z t+c \operatorname{tr} A B D^{-1}+d \operatorname{tr} A B C^{-1}-z c d-\operatorname{tr} B^{-1} A^{-1} D C \\
& =z t+c\left(z d-x_{8}\right)+d\left(z c-x_{7}\right)-z c d-\operatorname{tr} B^{-1} A^{-1} D C \\
& =z t+c d z-d x_{7}-c x_{8}-\operatorname{tr} B^{-1} A^{-1} D C .
\end{aligned}
$$

Substituting these into the next equation obtained from $B^{-1} A^{-1} D C=$ $A^{-1} \cdot B^{-1} \cdot C D$ and (I3),

$$
\begin{aligned}
\operatorname{tr} B^{-1} A^{-1} D C= & a \operatorname{tr} B^{-1} C D+b \operatorname{tr} A^{-1} C D+z t-a b t-\operatorname{tr} B^{-1} A^{-1} C D \\
= & a\left(b t-x_{9}\right)+b(a t+w)+z t-a b t \\
& -z t-c d z+d x_{7}+c x_{8}+\operatorname{tr} B^{-1} A^{-1} D C
\end{aligned}
$$

we obtain

$$
d x_{7}+c x_{8}-a x_{9}=-a b t-b w+c d z .
$$

(7) From $B^{-1} C D C^{-1}=\operatorname{tr} A B^{-1} A^{-1} D, \operatorname{tr} B^{-1}\left(C D C^{-1}\right)$ equals

$$
\begin{aligned}
\operatorname{tr} A B^{-1} A^{-1} D & =\operatorname{tr} B \operatorname{tr} A A^{-1} D-\operatorname{tr} A B A^{-1} D=b d-\operatorname{tr} D A B A^{-1} \\
& =b d-(\operatorname{tr} B \operatorname{tr} D-\operatorname{tr} B D-\operatorname{tr} B A \operatorname{tr} A D+\operatorname{tr} A \operatorname{tr} A B D) \\
& =x_{6}+z x_{4}-a x_{8} .
\end{aligned}
$$

Here we have used (I2) and (2.1c). Then from (2.11),

$$
t x_{5}+a x_{8}-c x_{9}=z x_{4} .
$$

(8) From $B A^{-1} B^{-1} C=A^{-1} D C D^{-1}$ and (I2), we have

$$
a c-\operatorname{tr} B A B^{-1} C=\operatorname{tr} B A^{-1} B^{-1} C=\operatorname{tr} A^{-1} D C D^{-1}=a c-\operatorname{tr} A D C D^{-1} \text {, }
$$

and hence $\operatorname{tr} C B A B^{-1}=\operatorname{tr} A D C D^{-1}$. We have by using (2.1c)

$$
\begin{aligned}
\operatorname{tr} C B A B^{-1}= & \operatorname{tr} C \operatorname{tr} A-\operatorname{tr} A C-\operatorname{tr} B C \operatorname{tr} A B+\operatorname{tr} B \operatorname{tr} C B A \\
= & a c-x_{3}-z x_{5}+b(\operatorname{tr} C \operatorname{tr} B A+\operatorname{tr} B \operatorname{tr} C A+\operatorname{tr} A \operatorname{tr} C B \\
& -\operatorname{tr} A \operatorname{tr} B \operatorname{tr} C-\operatorname{tr} A B C) \\
= & a c-x_{3}-z x_{5}+b c z+b^{2} x_{3}+a b x_{5}-a b^{2} c-b x_{7}
\end{aligned}
$$

and

$$
\begin{aligned}
\operatorname{tr} A D C D^{-1}= & \operatorname{tr} A \operatorname{tr} C-\operatorname{tr} A C-\operatorname{tr} A D \operatorname{tr} D C+\operatorname{tr} D \operatorname{tr} A D C \\
= & a c-x_{3}-t x_{4}+d(\operatorname{tr} A \operatorname{tr} C D+\operatorname{tr} D \operatorname{tr} A C+\operatorname{tr} C \operatorname{tr} A D \\
& -\operatorname{tr} A \operatorname{tr} D \operatorname{tr} C-\operatorname{tr} A C D) \\
= & a c-x_{3}-t x_{4}+a d t+d^{2} x_{3}+c d x_{4}-a d^{2} c+w d .
\end{aligned}
$$

Thus we obtain

$(z-a b) x_{5}+b x_{7}=\left(b^{2}-d^{2}\right) x_{3}+(t-c d) x_{4}+b c z-a b^{2} c-a d t+a d^{2} c-w d$. 
(9) We use $C^{-1} B A=\operatorname{tr} D C^{-1} D^{-1} A B$. Then from (I2) and (I3),

$$
\begin{aligned}
\operatorname{tr} C^{-1} B A & =z c-\operatorname{tr} C B A \\
& =z c-\left(c z+b x_{3}+a x_{5}-a b c-x_{7}\right)=-b x_{3}-a x_{5}+a b c+x_{7} .
\end{aligned}
$$

From (I2) and (2.1c) this equals

$$
\begin{aligned}
\operatorname{tr}\left(D C^{-1} D^{-1}\right) A B= & c z-\operatorname{tr} A B D C D^{-1} \\
= & c z-(\operatorname{tr} A B \operatorname{tr} C-\operatorname{tr} A B C-\operatorname{tr} A B D \operatorname{tr} C D \\
& +\operatorname{tr} D \operatorname{tr}(A B \cdot D \cdot C)) \\
= & x_{7}+t x_{8}-d\left(z t+d x_{7}+c x_{8}-z c d-x_{10}\right) .
\end{aligned}
$$

Hence we obtain

$$
-a x_{5}+d^{2} x_{7}+(c d-t) x_{8}-d x_{10}=-a b c+b x_{3}-d t z+c d^{2} z .
$$

(10) We use $D^{-1} C^{-1} B=C^{-1} D^{-1} A B A^{-1}$. From (I2), $\operatorname{tr} D^{-1} C^{-1} B=$ $b t-x_{9}$ and from (I2), (2.1c) and (I3),

$$
\begin{aligned}
\operatorname{tr} C^{-1} D^{-1} A B A^{-1}= & t b-\operatorname{tr}(D C) A B A^{-1} \\
= & t b-(t b-\operatorname{tr} D C B-\operatorname{tr} D C A \operatorname{tr} A B+\operatorname{tr} A \operatorname{tr}(D \cdot C \cdot A B)) \\
= & \left(d x_{5}+c x_{6}+b t-b c d-x_{9}\right)+z\left(d x_{3}+c x_{4}+a t-a c d+w\right) \\
& -a\left(z t+d x_{7}+c x_{8}-z c d-x_{10}\right)
\end{aligned}
$$

we obtain

$$
d x_{5}+c x_{6}-a d x_{7}-a c x_{8}+a x_{10}=b c d-z d x_{3}-z c x_{4}-z w .
$$

Let

$$
M=\left(\begin{array}{cccccc}
d t-c & d & 0 & 0 & -t & 0 \\
0 & 0 & d & c & -a & 0 \\
t & 0 & 0 & a & -c & 0 \\
z-a b & 0 & b & 0 & 0 & 0 \\
-a & 0 & d^{2} & c d-t & 0 & -d \\
d & c & -a d & -a c & 0 & a
\end{array}\right), \vec{x}=\left(\begin{array}{c}
x_{5} \\
x_{6} \\
x_{7} \\
x_{8} \\
x_{9} \\
x_{10}
\end{array}\right)
$$

and

$$
\vec{v}=\left(\begin{array}{c}
2 b-b t^{2}+b c d t-b c^{2} \\
-a b t-b w+c d z \\
z x_{4} \\
\left(b^{2}-d^{2}\right) x_{3}+(t-c d) x_{4}+b c z-a b^{2} c-a d t+a c d^{2}-w d \\
-a b c+b x_{3}-d z t+c d^{2} z \\
b c d-d z x_{3}-c z x_{4}-z w
\end{array}\right) .
$$


From the results (5)-(10) we obtain $M \vec{x}=\vec{v}$. The matrix $M$ is singular, if $a=c$. However, by using (2.4) and (2.7) we can deduce:

(2.12)

$$
\begin{aligned}
& x_{5}=\frac{c\left(2 b+a^{2} b-2 a z+b K^{2}\right)-t u z+d w\left(a b+z+z K^{2}\right)-v\left(a b+z K^{2}\right)}{K^{2}+a^{2}}, \\
& x_{6}=\frac{2(a d z-b d)-u\left(a b+K^{2} z\right)+t v\left(a b+z+K^{2} z\right)+(c-d t) w\left(a b+z+K^{2} z\right)}{K^{2}+a^{2}}, \\
& x_{7}=\frac{-2 c z-b t u+a v z+w d(b-a z)}{K^{2}+a^{2}}, \\
& x_{8}=\frac{d\left(K^{2}+a^{2}+2\right)+a u z+v t(b-a z)+w(b c-b d t-a c z+a d t z)}{K^{2}+a^{2}}, \\
& x_{9}=\frac{t\left(2 b+a^{2} b-2 a z+b K^{2}\right)+d v z+w\left(a b+K^{2} z\right)+u(c z-d t z)}{K^{2}+a^{2}}, \\
& x_{10}=\frac{-2 t z+b(c-d t) u+b d v-a w z}{K^{2}+a^{2}} .
\end{aligned}
$$

Expressions for $x_{3}$ and $x_{4}$ are obtained in (2.10).

3. Mapping class group. Let $G$ be a group of type $(2 ;-;-;-)$ and $E=$ $(A, B, C, D)$ a marking (or a canonical generator system) of $G$. We consider the following changes of marking:

$$
\begin{array}{ll}
\omega_{1}(E)=\left(A B^{-1}, B, C, D\right), & \omega_{2}(E)=(B, B A, C, D), \\
\omega_{3}(E)=\left(B^{-1} C A, B, C, B^{-1} C D\right), & \\
\omega_{4}(E)=\left(A, B, C D^{-1}, D\right), & \omega_{5}(E)=(A, B, C, D C) .
\end{array}
$$

Each $\omega_{j}$ induces an automorphism of $G$, which is also denoted by $\omega_{j}$. The table below shows the images of the elements in the leftmost column un$\operatorname{der} \omega_{j}$.

\begin{tabular}{|c||c|c|c|c|c|}
\hline & $\omega_{1}$ & $\omega_{2}$ & $\omega_{3}$ & $\omega_{4}$ & $\omega_{5}$ \\
\hline$A$ & $A B^{-1}$ & $A$ & $B^{-1} C A$ & $A$ & $A$ \\
\hline$B$ & $B$ & $B A$ & $B$ & $B$ & $B$ \\
\hline$A B$ & $A$ & $A B A$ & $B^{-1} C A B$ & $A B$ & $A B$ \\
\hline$A C D C^{-1}$ & $A B^{-1} C D C^{-1}$ & $A C D C^{-1}$ & $B^{-1} C A C B^{-1} C D C^{-1}$ & $A C D C^{-1}$ & $A C D$ \\
\hline$A C D^{2}$ & $A B^{-1} C D^{2}$ & $A C D^{2}$ & $B^{-1} C A C\left(B^{-1} C D\right)^{2}$ & $A C D$ & $A C(D C)^{2}$ \\
\hline$A C D$ & $A B^{-1} C D$ & $A C D$ & $B^{-1} C A C B^{-1} C D$ & $A C$ & $A C D C$ \\
\hline$C D$ & $C D$ & $C D$ & $C B^{-1} C D$ & $C$ & $C D C$ \\
\hline \multicolumn{5}{|c|}{}
\end{tabular}


Let $\omega_{j *} \in \mathcal{M} C_{2}$ denote the mapping class induced by $\omega_{j}$. Then $\omega_{1 *}, \ldots$, $\omega_{5 *}$ generate $\mathcal{M} C_{2}$ and satisfy the following relations [1, Theorem 4.8]:

$$
\begin{gathered}
\omega_{i *} \omega_{j *}=\omega_{j *} \omega_{i *} \text { if }|i-j| \geq 2,1 \leq i, j \leq 5, \\
\omega_{j *} \omega_{j+1 *} \omega_{j *}=\omega_{j+1 *} \omega_{j *} \omega_{j+1 *}(j=1,2,3,4), \\
\left(\omega_{1 *} \omega_{2 *} \omega_{3 *} \omega_{4 *} \omega_{5 *}\right)^{6}=1, \\
\omega_{1 *} \omega_{2 *} \omega_{3 *} \omega_{4 *} \omega_{5 *}^{2} \omega_{4 *} \omega_{3 *} \omega_{2 *} \omega_{1 *}=1 .
\end{gathered}
$$

In this section we represent the action of $\omega_{j *}$ on $\mathcal{T}_{2}$ in the variables $a, b, z, u, v$, $w, t$. More precisely, when $\left(A_{j}, B_{j}, C_{j}, D_{j}\right)=\omega_{j}(A, B, C, D)$, we express

$$
\begin{array}{lll}
a_{j}=\operatorname{tr} A_{j}, & b_{j}=\operatorname{tr} B_{j}, & z_{j}=\operatorname{tr} A_{j} B_{j}, \\
v_{j}=-\operatorname{tr} A_{j} C_{j} D_{j}^{2}, & w_{j}=-\operatorname{tr} A_{j} C_{j} D_{j}, & t_{j}=\operatorname{tr} A_{j} C_{j} D_{j} C_{j} D_{j}^{-1},
\end{array}
$$

by using $a, b, z, u, v, w, t$. However, for the case of $\omega_{3}$ we modify the signs of some traces to obtain positive values.

(Case of $\omega_{1 *}$ ) By using basic trace identities we have $\operatorname{tr} A B^{-1}=\operatorname{tr} A \operatorname{tr} B-$ $\operatorname{tr} A B=a b-z$,

$$
\begin{aligned}
& w_{1}=-\operatorname{tr} A B^{-1} C D=-\operatorname{tr} B \operatorname{tr} A C D+\operatorname{tr} A B C D=b w+x_{10}, \\
& u_{1}=-\operatorname{tr} A B^{-1} C D C^{-1}=-\operatorname{tr} B \operatorname{tr} A C D C^{-1}+\operatorname{tr}(A B) C D C^{-1} \quad(\because \text { (I2)) } \\
& =b u+(\operatorname{tr} A B \operatorname{tr} D-\operatorname{tr} A B D \\
& -\operatorname{tr} A B C \operatorname{tr} C D+\operatorname{tr} C \operatorname{tr} A B C D)(\because(2.1 c)) \\
& =b u+z d-x_{8}-t x_{7}+c x_{10},
\end{aligned}
$$

and

$$
\begin{aligned}
v_{1}=-\operatorname{tr} A B^{-1} C D^{2} & =-\operatorname{tr} B \operatorname{tr} A C D^{2}+\operatorname{tr} A B C D^{2} \quad(\because(\mathrm{I} 2)) \\
& =b v+(\operatorname{tr} A B C D \operatorname{tr} D-\operatorname{tr} A B C) \quad(\because(\mathrm{I} 2)) \\
& =b v+d x_{10}-x_{7}
\end{aligned}
$$

Hence

$$
\omega_{1 *}(a, b, z, u, v, w, t)=\left(a b-z, b, a, u_{1}, v_{1}, w_{1}, t\right) .
$$

(Case of $\omega_{2 *}$ ) Since $\operatorname{tr} A B A=\operatorname{tr} A B \operatorname{tr} A-\operatorname{tr} B=z a-b$,

$$
\omega_{2 *}(a, b, z, u, v, w, t)=(a, z, a z-b, u, v, w, t) .
$$

(Case of $\omega_{3 *}$ ) First we remark that $\operatorname{tr} B^{-1} C A<0$ and $\operatorname{tr} B^{-1} C D<0$. To see $\operatorname{tr} B^{-1} C A<0$, for example, note that $\left(A B^{-1}, B\right)$ is a marking for a group of type $(1 ; 0 ; 0 ; 1)$ and $\operatorname{tr} A$ and $\operatorname{tr} B$ are positive. Then we have $\operatorname{tr} A B^{-1}>0$. Then $\left(A B^{-1}, C\right)$ is a marking for a group of type $(0 ; 0 ; 0 ; 3)$. Since $\operatorname{tr} A B^{-1}$ and $\operatorname{tr} C$ are positive, $\operatorname{tr} A B^{-1} C<0$ (see [5, Section $33 \mathrm{~A}$ and $\mathrm{D}])$. The calculation for $\omega_{3 *}$ is the most complicated: By using the basic trace identities we have

$$
a_{3}=\operatorname{tr} B^{-1} C A=\operatorname{tr} B \operatorname{tr} A C-\operatorname{tr} A B C=b x_{3}-x_{7} .
$$




$$
\begin{aligned}
& w_{3}=-\operatorname{tr}\left(B^{-1} C\right)(A C)\left(B^{-1} C\right) D \\
& =-\operatorname{tr}(A C)\left(B^{-1} C\right) D\left(B^{-1} C\right) \\
& =-\operatorname{tr} A C B^{-1} C \operatorname{tr} B^{-1} C D-\operatorname{tr} A C D+\operatorname{tr} A C \operatorname{tr} D \\
& =-\left(\operatorname{tr} B \operatorname{tr} A C^{2}-\operatorname{tr} A C B C\right)(\operatorname{tr} B \operatorname{tr} C D-\operatorname{tr} B C D)+w+d x_{3} \\
& =-\left[b\left(c x_{3}-a\right)-\left(x_{3} x_{5}+z-a b\right)\right]\left(b t-x_{9}\right)+w+d x_{3} \\
& =\left(x_{3} x_{5}+z-b c x_{3}\right)\left(b t-x_{9}\right)+w+d x_{3} \text {, } \\
& u_{3}=-\operatorname{tr}\left(B^{-1} C\right)(A C)\left(B^{-1} C\right)\left(D C^{-1}\right) \\
& =-\operatorname{tr}(A C)\left(B^{-1} C\right)\left(D C^{-1}\right)\left(B^{-1} C\right) \\
& =-\operatorname{tr} A C B^{-1} C \operatorname{tr} B^{-1} C D C^{-1}-\operatorname{tr} A C D C^{-1}+\operatorname{tr} A C \operatorname{tr} D C^{-1} \\
& =-\left(\operatorname{tr} A C \operatorname{tr} B^{-1} C-\operatorname{tr} A B\right)\left(\operatorname{tr} B \operatorname{tr} D-\operatorname{tr} B C D C^{-1}\right)+u+x_{3}(c d-t) \\
& =-\left(x_{3}\left(b c-x_{5}\right)-z\right)\left[b d-\left(b d-x_{6}-t x_{5}+c x_{9}\right)\right]+u+x_{3}(c d-t) \\
& =\left(x_{3} x_{5}+z-b c x_{3}\right)\left(x_{6}+t x_{5}-c x_{9}\right)+u+x_{3}(c d-t) \text {, } \\
& v_{3}=-\operatorname{tr} B^{-1} C A C\left(B^{-1} C D\right)^{2} \\
& =-\operatorname{tr} B^{-1} C D \operatorname{tr} B^{-1} C A C B^{-1} C D+\operatorname{tr} B^{-1} C A C \\
& =\left(b t-x_{9}\right)\left[\left(x_{3} x_{5}+z-b c x_{3}\right)\left(b t-x_{9}\right)+w+d x_{3}\right]+\left(b c-x_{5}\right) x_{3}-z \text {, } \\
& t_{3}=\operatorname{tr} C B^{-1} C D=\operatorname{tr} C B^{-1} \operatorname{tr} C D-\operatorname{tr} B D=\left(b c-x_{5}\right) t-x_{6} .
\end{aligned}
$$

In this case $a_{3}, x_{3}, v_{3}$ and $t_{3}$ are negative. We modify the sign of these parameters and obtain

$$
\omega_{3 *}(a, b, z, u, v, w, t)=\left(-a_{3}, b,-x_{3}, u_{3},-v_{3}, w_{3},-t_{3}\right) .
$$

(Case of $\omega_{4 *}$ ) For the expression of $\omega_{4 *}$ we have easily

$$
\omega_{4 *}(a, b, z, u, v, w, t)=\left(a, b, z, u, w,-x_{3}, c\right) .
$$

(Case of $\left.\omega_{5 *}\right)$ Since $-\operatorname{tr} A C D C=-\operatorname{tr} C \operatorname{tr} A C D+\operatorname{tr} A C D C^{-1}=c w-u$,

$$
\begin{aligned}
v_{5} & =-\operatorname{tr} A C(D C)^{2}=-\operatorname{tr} C D \operatorname{tr} A C D C+\operatorname{tr} A C \\
& =-t\left(\operatorname{tr} C \operatorname{tr} A C D-\operatorname{tr} A C D C^{-1}\right)+x_{3} \\
& =c w t-t u+x_{3},
\end{aligned}
$$

and $\operatorname{tr} C D C=c t-d$, we have

$$
\omega_{5 *}(a, b, z, u, v, w, t)=\left(a, b, z, w, c w t-t u+x_{3}, c w-u, c t-d\right) .
$$

Now we conclude 
Theorem 3.1. The mapping classes $\omega_{1 *}, \omega_{2 *}, \omega_{3 *}, \omega_{4 *}, \omega_{5 *}$ are represented by the following rational maps in variables $a, b, z, u, v, w, t$ :

$$
\begin{aligned}
& \omega_{1 *}(a, b, z, u, v, w, t)=\left(a b-z, b, a, u_{1}, v_{1}, w_{1}, t\right) \\
& \omega_{2 *}(a, b, z, u, v, w, t)=(a, z, a z-b, u, v, w, t) \\
& \omega_{3 *}(a, b, z, u, v, w, t)=\left(-b x_{3}+x_{7}, b,-x_{3}, u_{3},-v_{3}, w_{3},-b c t+x_{5} t+x_{6}\right) \\
& \omega_{4 *}(a, b, z, u, v, w, t)=\left(a, b, z, u, w,-x_{3}, c\right) \\
& \omega_{5 *}(a, b, z, u, v, w, t)=\left(a, b, z, w, c w t-t u+x_{3}, c w-u, c t-d\right),
\end{aligned}
$$

where $c, d, x_{3}, x_{4}, x_{5}, x_{6}$ and $x_{7}$ are given in (2.9) and (2.10) and (2.12).

As it is shown in Section $2, x_{1}=c, x_{2}=d, \ldots, x_{10}$ are all rational functions in $(a, b, z, u, v, w, t)$. Hence the inverse mappings of $\omega_{j *}(j=1, \ldots, 5)$ are also rational mappings. The expressions in (3.2) in $(a, b, z, u, v, t)$, especially the one for $\omega_{3 *}$, are very complicated.

Acknowledgement. This paper is a development from the content of the second author's talk at the XVIth Conference on Analytic Functions and Related Topics held at Chełm during June 26-29, 2011. We thank the organizers, in particular, Professors J. Zając, B. Fałda and D. Partyka, for their invitation to the conference and hospitality. We also thank the referee for a careful reading and many useful suggestions.

\section{REFERENCES}

[1] Birman, J. S., Braids, links, and mapping class groups, Ann. of Math. Studies 82, Princeton Univ. Press, Princeton, N. J., 1974.

[2] Maclachlan, C., Reid, A. W., The Arithmetic of Hyperbolic 3-Manifolds, SpringerVerlag, New York, 2003.

[3] Nakamura, G., Nakanishi, T., Parametrizations of some Teichmüller spaces by trace functions, Conform. Geom. Dyn. 17 (2013), 47-57.

[4] Nakanishi, T., Näätänen, M., Parametrization of Teichmüller space by length parameters, Analysis and Topology (C. Andreian-Cazacu, O. Lehto and Th. M. Rassias, eds.), 541-560, World Sci. Publ., Singapore, 1998.

[5] Zieschang, H., Finite Groups of Mapping Classes of Surfaces, Springer-Verlag, Berlin, 1981.

Gou Nakamura

Science Division, Center for General Education

Aichi Institute of Technology

1247 Yachigusa, Yakusa, Toyota

470-0392, Japan

e-mail: gou@aitech.ac.jp

Toshihiro Nakanishi

Department of Mathematics, Shimane University

Matue, 690-8504, Japan

e-mail: tosihiro@riko.shimane-u.ac.jp

Received September 27, 2011 